\title{
Microscopic model for switching kinetics in organic ferroelectrics following the Merz law
}

\author{
Tim D. Cornelissen $\odot,{ }^{1,2}$ Indre Urbanaviciute $\odot,{ }^{1}$ and Martijn Kemerink $\oplus^{1,2, *}$ \\ ${ }^{1}$ Complex Materials and Devices, Department of Physics, Chemistry and Biology (IFM), Linköping University, 58183 Linköping, Sweden \\ ${ }^{2}$ Centre for Advanced Materials, Ruprecht-Karls-Universität Heidelberg, Im Neuenheimer Feld 225, 69120 Heidelberg, Germany
}

(Received 28 February 2020; revised manuscript received 24 April 2020; accepted 14 May 2020;

published 1 June 2020)

\begin{abstract}
From an application perspective, one of the most important parameters of a ferroelectric is its switching time, and understanding its limiting factors is key to improve device performance. While there is a variety of competing models for switching kinetics in realistic (disordered) ferroelectrics, they are often merely descriptive and provide little insight into the underlying microscopic mechanisms. This holds in particular for the classical Merz law, which describes the commonly observed exponential field dependence of the switching time. Here, we investigate the switching kinetics in the archetypical molecular ferroelectric trialkylbenzene-1,3,5tricarboxamide using an electrostatic kinetic Monte Carlo model. The simulated field dependence follows the Merz law, which shows that a simple system of interacting dipoles is sufficient to obtain this behavior, even without explicitly considering domain walls or defects that are commonly thought to be involved in the emergence of the Merz law. Through a detailed analysis of the nucleation process, we can relate the macroscopic switching time to the microscopic nucleation energy barrier, which in turn is related to a field-dependent nucleus size. Finally, we use the acquired insight into the nucleation process to derive the Merz law from the theory of thermally activated nucleation-limited switching. This analytical model provides a physically transparent description of the switching kinetics in both experiments and simulations.
\end{abstract}

DOI: 10.1103/PhysRevB.101.214301

\section{INTRODUCTION}

The speed of polarization reversal is an important parameter for ferroelectric applications. It determines the maximal operating frequency of ferroelectric devices such as memories. While the switching speed in inorganic ferroelectrics such as lead zirconate titanate and barium titanate typically goes down to the order of several nanoseconds [1-3], or even subnanoseconds [4], organic ferroelectrics quite literally lag behind. For example, the ferroelectric copolymer P(VDFTrFE) typically switches on the order of several microseconds [5-7], although that can be pushed down to nanoseconds for very high fields applied on very thin films [8]. A good understanding of the switching kinetics is paramount if the switching speeds are to be increased further.

A multitude of competing models describing switching kinetics in ferroelectric materials exist. Classically, the Kolmogorov-Avrami-Ishibashi (KAI) model is used, which is based on polarization reversal through nucleation and growth [9-11]. The change in polarization is given by

$$
\Delta P(t)=2 P_{r}\left(1-\exp \left[-(t / \tau)^{n}\right]\right),
$$

\footnotetext{
*martijn.kemerink@cam.uni-heidelberg.de
}

Published by the American Physical Society under the terms of the Creative Commons Attribution 4.0 International license. Further distribution of this work must maintain attribution to the author(s) and the published article's title, journal citation, and DOI. Funded by Bibsam. where $P_{r}$ is the remanent polarization, $\tau$ the characteristic switching time, and $n$ the dimensionality of the domain growth. While applicable to bulk single crystals, the KAI model fails to describe the switching dynamics in polycrystalline, thin film, or organic ferroelectrics [12-15].

The switching time typically decreases when the temperature $T$ or the applied electric field $E$ are increased. The latter dependence is usually described by the semiempirical Merz law [16]:

$$
\tau=\tau_{\infty} \exp \left(\frac{E_{a}}{E}\right)
$$

where $\tau_{\infty}$ is the switching time at infinite applied field, and $E_{a}$ the so-called activation field, both of which typically depend on temperature, although a variety of functional dependencies has been observed $[1,5,6,17,18]$. While this description works over a remarkable range of materials, electric fields, and timescales, several deviations have been observed $[1,8,19$ 22]. Furthermore, as an empirical law it provides no insight into the underlying mechanisms of switching. Theoretical and experimental efforts to uncover the physical origin of the activation field have related it to nucleation and domain-wall depinning [17,18,23-26], but it is not always evident how universally valid those insights are and how they translate to, e.g., organic ferroelectrics. For the liquid crystalline organic ferroelectric trialkylbenzene-1,3,5- tricarboxamide (BTA), significant deviations from the Merz law were observed [19]. Similar deviations were found for the polymer ferroelectric $\mathrm{P}(\mathrm{VDF}: \operatorname{TrFE})[8,22]$.

Due to the failure of the KAI and Merz models to describe disordered ferroelectrics, and the lack of physical insight they 

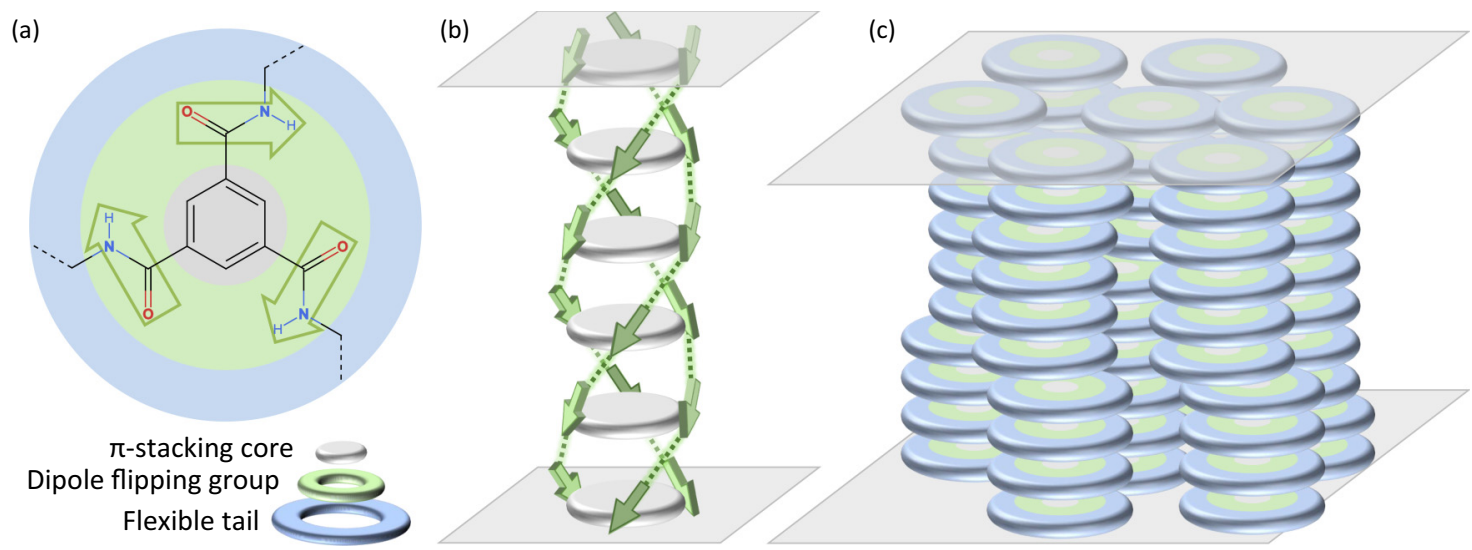

FIG. 1. Morphology of the BTA system. (a) The BTA molecule consists of a benzene core, three dipolar amide groups, and flexible alkyl tails. (b) The molecules stack into columns, forming a triple hydrogen-bonded helix. (c) A real device consists of hexagonally packed columns with defects that introduce disorder.

provide, several more advanced theories have been developed. One of these is the nucleation-limited switching (NLS) model by Tagantsev et al. [1,12], which essentially introduces a distribution of switching times into the KAI formalism. Zhukov et al. came to a similar result but provided a physical basis for this distribution with their inhomogeneous field mechanism (IFM) model [3,27]. Vopsaroiu et al. took a more fundamental approach starting from the Landau free-energy landscape to derive a theory of thermally activated nucleation limited switching (TA-NLS) [28]. This model allows one to extract microscopic parameters but is at odds with the Merz law in that it predicts the switching time to scale as $\tau \propto \exp (-E)$. Recently, we have combined several of these models to describe the shape of the dispersive switching transients in organic ferroelectrics based on their particular morphology [29].

Here we investigate the field dependence of the switching kinetics in organic ferroelectrics using a combination of analytical and microscopic modeling. With the previously introduced electrostatic Monte Carlo model we simulate switching transients in the molecular ferroelectric BTA [30]. The field dependence of the switching time is found to follow the Merz law, showing that Merz-type behavior can arise in a simple system of interacting dipoles. We can directly relate this behavior to the microscopic nucleation process. This allows us to construct a physically transparent analytical description of the switching kinetics.

\section{A. Electrostatic kinetic Monte Carlo model}

Our model system is the prototypical molecular ferroelectric BTA. The hierarchical morphology of this material is shown in Fig. 1, which shows the formation of supramolecular columns that organize into a hexagonal liquid crystal. The ferroelectricity in this material is caused by the dipolar amide groups that align and form a hydrogen-bonded triple-helix structure. By applying an electric field, the dipoles can be flipped and thereby the polarization reverses. We have recently shown BTA to be truly ferroelectric [19], and that its ferroelectric properties can easily be tuned by modifying the molecular structure [31,32].
The model we will use to study the polarization switching in BTA is described in detail in a previous work [30] and in section 1 of the Supplemental Material (SM) [33], and we will therefore only give a brief overview here. As the ferroelectricity in BTA stems from the dipolar amide groups, we focus solely on these dipoles and their electrostatic interactions. The positions of the dipoles are fixed and determined by the morphology of BTA. Using simple electrostatic calculations, we determine the energy it would cost to flip a certain dipole and convert this into a flipping rate using a Boltzmann factor. The flipping rates of all dipoles are the input for a kinetic Monte Carlo simulation, which results in a real-time evolution of the polarization in the material.

In previous works, we have shown the large influence structural disorder can have on the properties of BTA derivatives. In simulations, an increase in disorder was associated with a deterioration of the ferroelectric properties, especially the retention time [30]. Experimental results show a similar trend [31,34]. In contrast, the (negative) piezoelectric coefficient was found to increase in magnitude with increasing disorder [35]. We can introduce this disorder into our model by having defects that represent a break in the hydrogen-bonded triple helix, dividing the quasi-one-dimensional (1D) columns into subcolumns. Each defect will introduce a translational and rotational offset, and possibly a change in helicity.

\section{RESULTS AND DISCUSSION}

\section{A. Field dependence of switching time}

We first simulate the switching transients, i.e., the polarization response to a step in the applied electric field, using a similar configuration as in our previous work [30]. The switching time is taken as the point in the switching transient where the polarization crosses zero. In Fig. 2 the results (blue triangles) are compared to representative experiments for BTA. Although we do reproduce the functional Merz shape of Eq. (2) in the simulations, we find that the switching is either too fast at high fields, or too dispersive at low fields, as shown in detail in Fig. S2 [33]. Furthermore, Fig. 2 shows that the field dependence of the switching time is much stronger in the simulations than in the experiments. It should be noted that in 


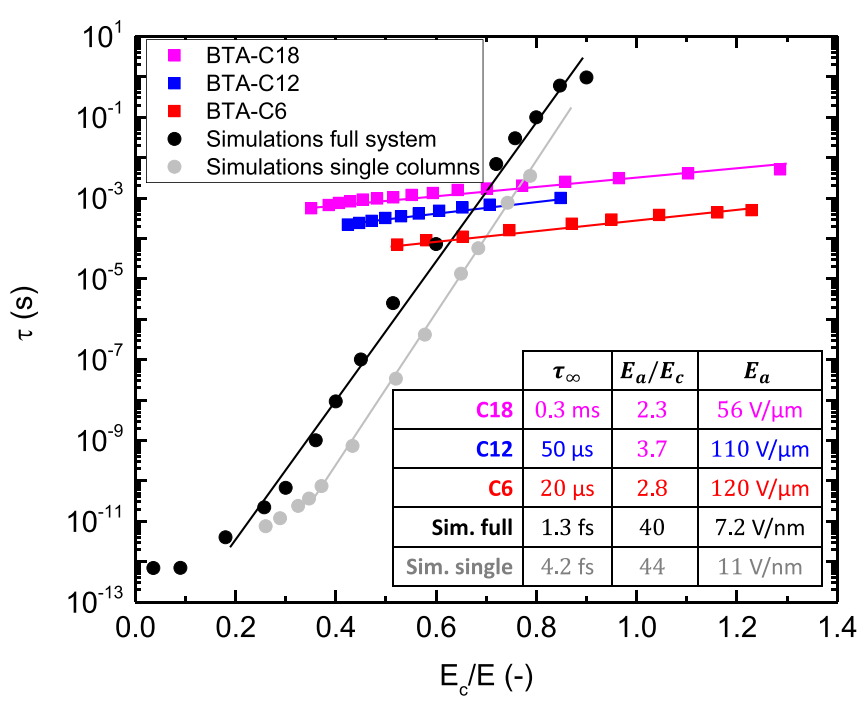

FIG. 2. The switching time $\tau$ as a function of the reciprocal applied field $1 / E$ normalized to the coercive field $E_{c}$ (at $1 \mathrm{~Hz}$ ). Data for the three BTA homologues $\mathrm{C} 6, \mathrm{C} 12$, and $\mathrm{C} 18$ are taken from Ref. [31]. Solid lines are fits to the Merz law of Eq. (2) with the indicated parameters.

other ferroelectrics, the normalized slope $E_{a} / E_{c}$ is generally between 10 and 40 , i.e., close to the simulated value of 40 , suggesting that BTA is a unique system with a low normalized activation field around 3 [23].

Before analyzing the data in Fig. 2 in terms of a fielddependent nucleation barrier and volume, and accordingly coming to an improved description of the experimental data, we ruled out several alternative explanations, full details of which are given in Appendixes A and B. In short, Appendix A shows that introducing different types of defects (chiral and achiral) in the simulations cannot remove the discrepancy with experiment, nor does introducing intercolumn interactions. Appendix B shows that neither the inclusion of "dead" interfacial layers, being highly disordered or even non-switching, can resolve the issue. Likewise, interpretations of the experiments in terms of the "leaky layer" model by von Seggern and Fedosov or in terms of system $R C$ times are ruled out $[36,37]$.

A reason for the discrepancy could be the finite dipole switching time in the actual material. There, the reversal of one dipole requires a physical rotation of the amide group and its attached alkyl chain, which is hindered by steric interactions and takes a finite amount of time. However, in our numerical simulations dipoles flip instantaneously, and such a reversal time is thereby ignored. The total switching time is still limited by the attempt frequency, which determines how often a flipping event can be initiated per unit time, but this is fundamentally different from the reversal time, which determines how long such a flipping event, once initiated, takes. It is unfortunately not possible to incorporate this in the kMC model, as this would require multiple events to take place at the same time. It is not unreasonable to assume that such a reversal time would not only reduce the absolute values, but also the field-dependence of the switching time, as especially at higher fields, where switching initiates faster, the response will be slowed down by the reversal time.

\section{B. Merz law and nucleation}

Both the experimental and the simulated results in Fig. 2 fit well to the functional form of the Merz law, even if the slopes do not match. The experiments show a slight deviation towards faster switching at higher fields, as has been observed before [19]. The simulations show a different deviation at high fields, where the switching time no longer decreases. At these fields $\left(E_{c} / E<0.1\right)$, switching is no longer stochastic nor nucleation limited, with all dipoles switching immediately. The switching time is then limited by the attempt frequency $v_{0}$ that is set to $1 \mathrm{THz}$ in the simulations.

We would like to emphasize the fact that this simple system of interacting dipoles follows the Merz law. The origin of the Merz law is typically given as creep behavior of the ferroelectric domain walls in a pinning potential or, equivalently, as nucleation of a reversed domain [17,18,25,26,38]. Following classical nucleation theory one can construct an energy balance between bulk and surface energies of the nucleus. From this the correct $1 / E$ scaling of the energy barrier for nucleation can be obtained. However, this derivation is highly dependent on the assumed geometry of the nucleus $[17,18,23,24]$.

In our case, Merz behavior emerges from a collection of purely electrostatically interacting dipoles, without explicitly including terms like domain wall or depolarization energy. Furthermore, it is also obtained in a system without any defects that could provide pinning sites, and even in an infinitely long column without contacts, see Fig. S3 [33]. This agrees with simulations from Leschorm et al., who observed similar behavior from a slightly different electrostatic model, although Merz behavior was there found only over a smaller range of fields $[39,40]$. This shows that the Merz law is more general than previously assumed.

Previously, we have shown that the polarization switching in BTA is nucleation limited in both experiments and simulations [19,30,31]. To investigate the nucleation process and its relation to the Merz law in detail, we continue by simulating individual columns without defects. This change from a box of interacting columns to single-column simulations speeds up calculations and simplifies the analysis. That this change is justified is demonstrated by the gray points in Fig. 2; the switching times of the single columns are close to that of the full box and show the same functional dependence. Section 3 of the SM and the corresponding videos further show that the interaction between columns can be neglected when investigating the nucleation mechanism [33].

In a single column without defects, the contacts at the top and bottom of the material will be the preferred nucleation sites. Aside from the switching time we also track the nucleation process and directly obtain information about the energy and size of the nucleus. An example of a typical nucleation process is given in Fig. 3. From the energy as a function of time in Fig. 3(a) we can obtain the critical energy barrier $U^{*}$ as well as the critical nucleus size $N^{*}$.

We perform this analysis for ensembles of columns at different temperatures and the results are shown in Fig. 4. Not only the switching time [Fig. 4(a)] but also the energy barrier [Fig. 4(c)] and nucleus size [Fig. 4(d)] are proportional to $1 / E$ for low temperatures. We therefore analyze these results using 

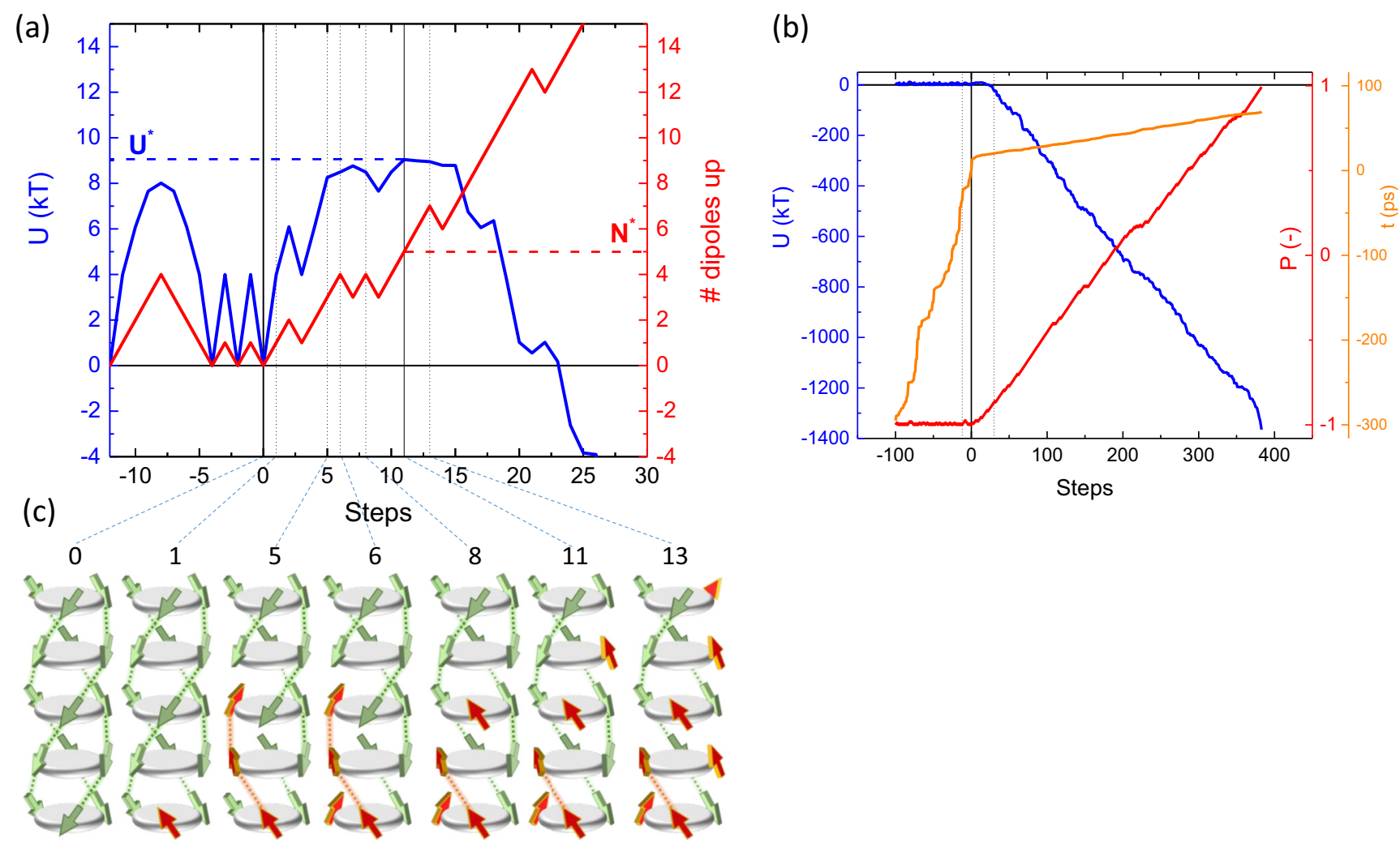

FIG. 3. An example of the nucleation process in a column without defects. (a) The energy and polarization throughout the nucleation phase. Steps are defined relative to the start of the nucleation at step 0 . The critical nucleus size and energy, occurring at step 11, are indicated. (b) The energy, polarization, and time throughout the subsequent steps in the entire switching process, where the steps shown in (a) are indicated. (c) Schematic view of the nucleation corresponding to the indicated steps in (a).

a more general form of the Merz law in Eq. (2):

$$
\begin{aligned}
\tau(E, T) & =\tau_{\infty} \exp \left(\frac{E_{a}(T)}{E}\right)=\tau_{\infty} \exp \left(\frac{U^{*}(E)}{k_{B} T}\right) \\
& =\tau_{\infty} \exp \left(\frac{U_{0}}{k_{B} T} \frac{E_{0}}{E}\right),
\end{aligned}
$$

with $U_{0}$ the field-independent energy barrier and $E_{0}$ a critical field. We fit the data in Fig. 4(b) to the temperature-dependent activation field $E_{a}(T)=U_{0} E_{0} / k_{B} T$. Note that a constant offset $E_{\text {offset }}=-3.9 \mathrm{~V} / \mathrm{nm}$ needs to be added to $E_{a}(T)$ to account for the activation field reaching zero already at a finite temperature. At this temperature the maximum switching speed of $1 \mathrm{THz}$ is obtained even for small fields. Similarly, we fit the data in Fig. 4(c) to the field-dependent nucleation energy barrier $U^{*}(E)=U_{0} E_{0} / E$ including an offset. Since we are mainly interested in the proportionality with field, both offsets are of minor importance for any of the subsequent

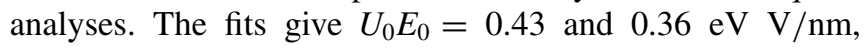
respectively, and the reasonable agreement between these two values quantitatively confirms the relation between the macroscopic switching time and the microscopic nucleation barrier. In other words, it suggests that it is the field dependence of the nucleation barrier that leads to the Merz behavior of the switching time.

The slight discrepancy between the two fits is likely caused by the oversimplification of considering the nucleation as a simple activation over an energy barrier. The prefactor $\tau_{\infty}$ as well as the fits in Fig. 4(c) are not fully temperature independent as Eq. (3) assumes, which we will discuss in more detail below. The pathway of nucleation is complex and involves several successive flips, some of which are upwards in energy. As can be seen in Fig. 3(c), dipoles can also flip back during the nucleation. Figure 3(c) also shows that the shape of the critical nucleus is not trivial and has a large surface area. This contrasts with most inorganic ferroelectrics, where the surface area of the nucleus is typically minimized resulting in a compact nucleus $[17,18,25]$.

The complex nature of the switching process also comes forward in the transition that is again visible around 0.63 $\mathrm{V} / \mathrm{nm}(\approx 1.6 \mathrm{~nm} / \mathrm{V})$ in Fig. 4(a), where the slope of the $\tau(1 / E)$ plot changes as in Fig. 2. The simulations allow us to see that this corresponds to a region where, at higher temperatures, the nucleation barrier and critical nucleus size no longer decrease with increasing applied field and can even increase again. We attribute this to more convoluted nucleation pathways becoming energetically accessible to the system at high fields and temperatures. At low fields and temperatures nucleation can only occur through a limited number of close to optimal pathways. In contrast, increasing the applied field decreases all energy barriers while increasing the temperature facilitates a type of thermal diffusion across the entire energy landscape. This combination leads to use of nonoptimal, in the meaning of shortest available, pathways. The result is an increase in the mean critical energy barrier and nucleus size, as well as an increased variation in those properties. 
(a)

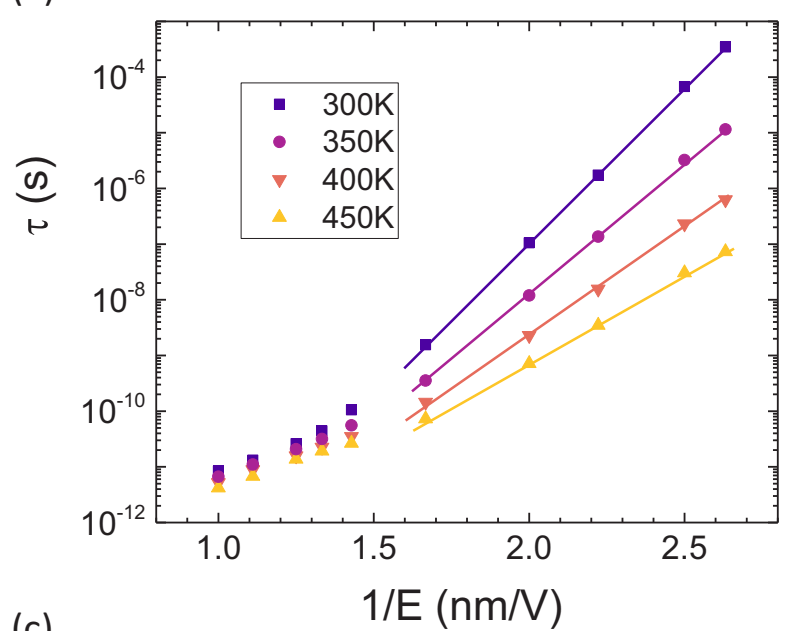

(c)

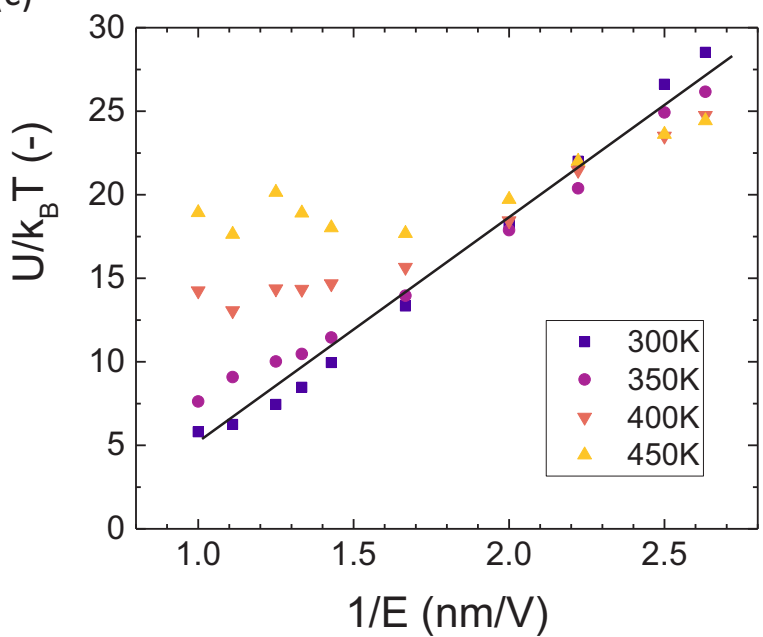

(b)

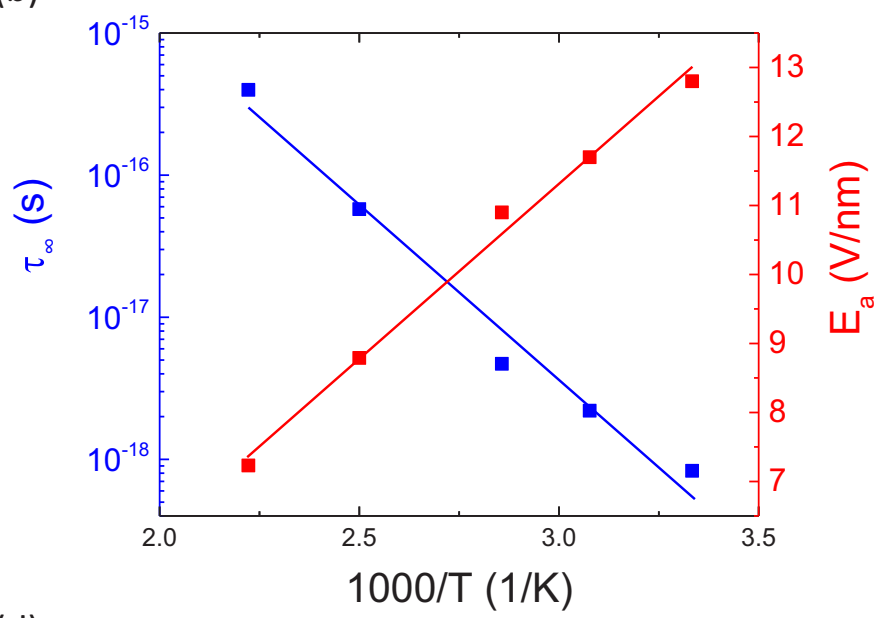

(d)

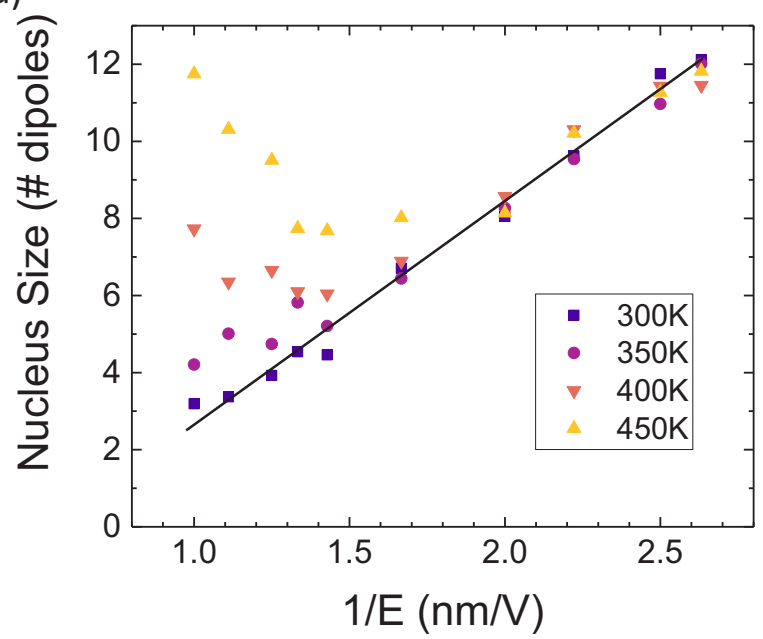

FIG. 4. Analysis of simulated switching transients at different temperatures. (a) Field dependence of switching time $\tau$ (symbols), lines are fits to Eq. (3). (b) Extracted parameters from the fits in (a), lines are fits to Eqs. (7) and (8). (c) Nucleation barrier $U^{*}$ (symbols), lines are fits to $U^{*}=U_{0} E_{0} / E$. (d) Size of the nucleus (symbols), lines are fits to Eq. (5). Results are averaged over 100 independent columns without defects.

\section{Thermally activated nucleation-limited switching}

Using the above, an insightful formulation of the Merz formula can be derived within the framework of thermally activated nucleation-limited switching developed by Vopsaroiu et al. [28]. As the name suggests, this framework regards ferroelectric switching as thermal activation over an energy barrier $W_{b}$. Within this framework an expression for both the coercive field as well as the switching time could be derived:

$$
\tau=\frac{1}{\nu_{0}} \exp \left(\frac{\left(w_{b}-P_{r} E\right) V^{*}}{k_{\mathrm{B}} T}\right),
$$

where $w_{B}$ is the energy barrier per unit volume, and $V^{*}$ the volume of the critical nucleus. The logarithmic switching time here is linearly dependent on the applied field, as opposed to inversely as follows from Merz. Depending on the range of applied fields, this can also give optically satisfying fits to the experimental switching times. However, such fits lead to material parameters that correspond to negative coercive fields at experimental timescales, i.e., they are unphysical.

The original theory of TA-NLS assumes a constant nucleation volume $V^{*}$, which is shown in Fig. 4(d) to be incor- rect. Instead we found this nucleation volume to be roughly inversely proportional to the applied field:

$$
V^{*}(E)=V_{0} N^{*}(E) \approx \frac{V_{0} E_{V}}{E},
$$

where $V_{0}$ is the effective volume of a single dipole and $E_{V}$ a characteristic field that corresponds to the slope of the fit in Fig. 4(d). Combining Eq. (5) with Eq. (4) we can modify the TA-NLS expression for the switching time:

$$
\tau=\frac{1}{v_{0}} \exp \left(\frac{-E_{V} V_{0} P_{r}}{k_{B} T}\right) \exp \left(\frac{w_{b} V_{0}}{k_{B} T} \frac{E_{V}}{E}\right) .
$$

By introducing the field-dependent nucleation volume, we have thus obtained a version of the Merz law from the theory of TA-NLS. This provides us with more transparent expressions for the mostly phenomenological parameters in Eq. (3):

$$
\begin{gathered}
\tau_{\infty}=\frac{1}{\nu_{0}} \exp \left(\frac{-E_{V} V_{0} P_{r}}{k_{B} T}\right), \\
E_{a}=\frac{w_{b} V_{0} E_{V}}{k_{B} T},
\end{gathered}
$$

and $U^{*}=U_{0} E_{0} / E$, where $U_{0}=w_{b} V_{0}$ and $E_{0}=E_{V}$. 
(a)

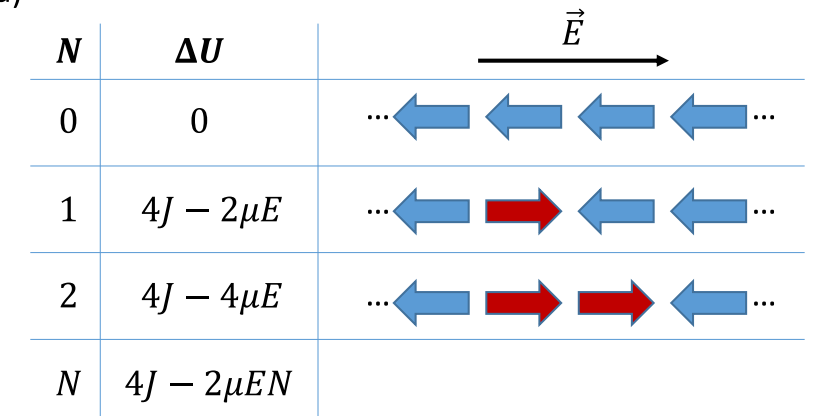

(b)

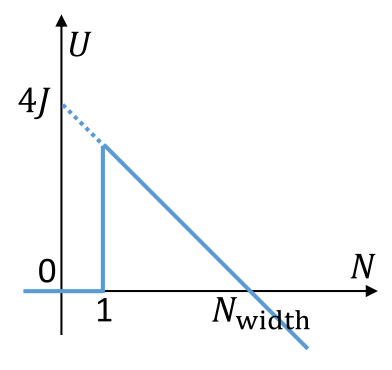

FIG. 5. Energy as a function of nucleus size in a simple 1D system of dipoles, shown (a) schematically and (b) graphically.

The energy-barrier density $w_{b}$ can independently be obtained from the frequency and temperature dependency of the coercive field and was previously found to be around $0.18 \mathrm{eV} / \mathrm{nm}^{3}[30,31]$. The effective volume $V_{0}$ is determined by the morphology of the system and calculated to be around $0.4 \mathrm{~nm}^{3}$. Using $E_{V}$ from Fig. 4(d) we find $w_{b} V_{0} E_{V} \approx$ $0.41 \mathrm{eV} \mathrm{V} / \mathrm{nm}$, which is in good agreement with the value for $U_{0} E_{0}$ obtained from the fits in Figs. 4(a) and 4(c).

Through Eq. (7) we also know the temperature dependence of the prefactor $\tau_{\infty}$, which is not explicitly included in the Merz law. We can fit this dependence to our simulation results for $\tau_{\infty}$ in Fig. 4(b). We get a perfect agreement using the same parameters used for the other fits. We find an effective attempt frequency of $v_{0} \approx 77 \mathrm{GHz}$ which is fairly low but reasonable.

The field-dependence of the switching time can thus be traced back to the field dependence of the nucleation mechanism and specifically the (variable) nucleus size. This dependence, quantified in Eq. (5), can be qualitatively understood by considering the width of the nucleation barrier, i.e., the number of switched dipoles from which point the energy becomes lower than the initial configuration. This width sets an upper limit for the nucleus size and makes for a more straightforward argument than the nucleus size itself.

For this argument we reduce the material to a collection of dipoles, as was done for the kMC model. If we consider only nearest-neighbor interactions and ignore intercolumnar interactions, we can describe the BTA columns as a quasi-1D stack of dipoles, or equivalently a 1D Ising chain. When an electric field is applied, a nucleus with dipoles that have aligned along the field direction may form. The energy of this system decreases linearly as a function of both nucleus size and applied field, as is shown in Fig. 5. The change in energy by aligning $N$ neighboring dipoles along the field direction is then $\Delta U=4 J-2 \mu E N$, where $J>0$ is the interaction energy between two neighboring dipoles. Setting $\Delta U=0$, this gives $N_{\text {width }}=J / \mu E$.

This simple argument thus gives a nucleus size inversely proportional to the field. While presented here for the $1 \mathrm{D}$ case, it can easily be extended to two-dimensional and three-dimensional systems where it results in similar expressions (see SM section 4 [33]). We would like to emphasize that while this intuitive argument rationalizes the $1 / E$ dependency, it is not meant to be a rigorous derivation of the nucleus energy and the simulation results are the main rationale behind introducing Eq. (5). In passing we note that any system with a linear dependence of energy on both field and length will show a similar $1 / E$-dependency, as for example in the well-known case of Fowler-Nordheim tunneling through a triangular barrier [41].

We can thus consistently describe the switching kinetics in our simulations using the adapted form of the TA-NLS theory of Eq. (6). We now turn back to the experiments. Figure 6 shows the switching time of BTA-C12, obtained using the experimental procedures described in Ref. [31]. We fit the data to Eq. (6) using a single parameter set for all curves and find a reasonable agreement over the full temperature and field range. While the individual experimental curves show deviations from Merz behavior, the overall behavior of the system is reproduced for reasonable parameters, again with the addition of an offset field $E_{\text {offset }}=-3.3 \mathrm{~V} / \mathrm{nm}$ to account for the effective vanishing of the activation barrier at finite temperatures. We find that $w_{b} V_{0} E_{V} \approx 0.10 \mathrm{eV} \mathrm{V} / \mathrm{nm}$, again showing that the field dependence, specifically the parameter $E_{V}$, is lower than in the simulations. A possible reason for that could be the existence of strong pinning defects in the

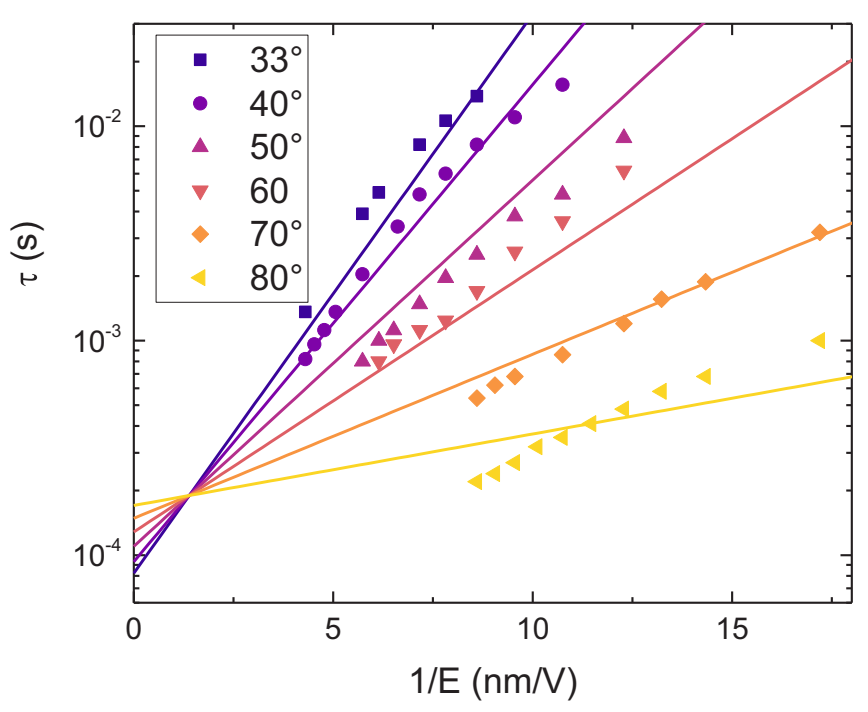

FIG. 6. The experimental switching time $\tau$ as a function of the reciprocal applied field $1 / E$ for a range of temperatures on BTA-C12. Straight lines are a global fit to Eq. (6). Details on the fit are presented in SM section 5 [33]. 
(a)

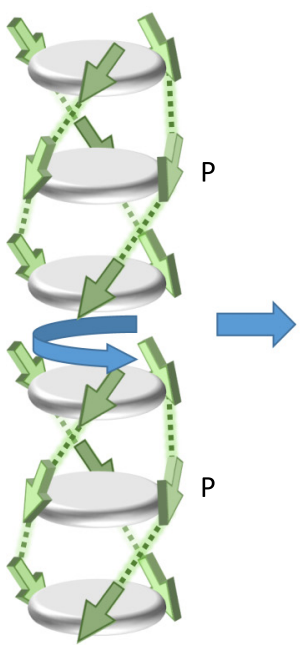

$\alpha= \pm 60^{\circ}$

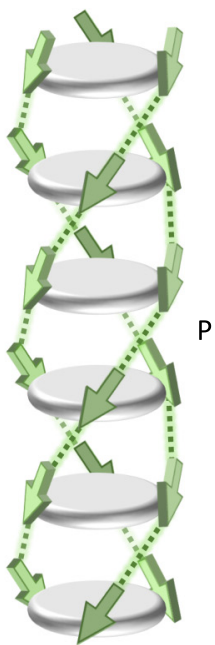

$\alpha=0^{\circ}$ (b)
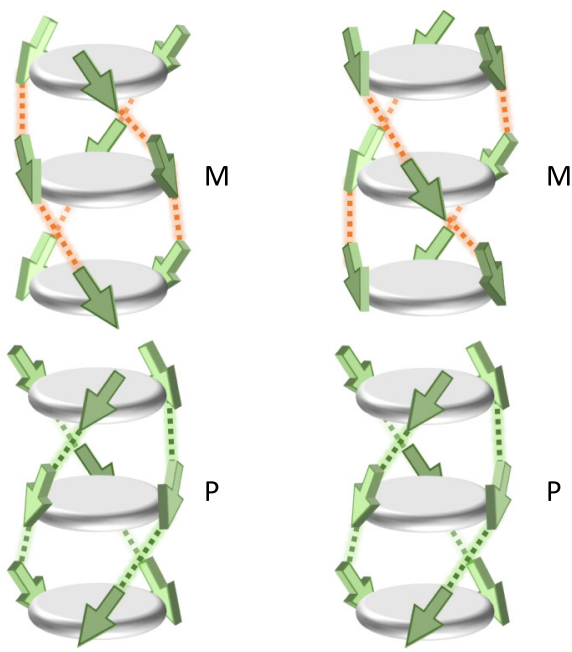

$\alpha= \pm 60^{\circ}$
M

FIG. 7. Single columns with defects that break the hydrogen-bonded triple helix. $\alpha$ denotes the rotational offset between the subcolumn below and above the defect. Defects can be (a) nonchiral and (b) chiral, where the helicity changes from $M$ to $P$. The nonchiral defect might easily rotate, which restores the triple helix, and thereby removes the defect. The chiral defect cannot be removed this way.

real material, which will restrict the nucleus size and thereby weaken the field dependency.

It should be noted that the prefactor $\tau_{\infty}$ is very sensitive to the exact parameters of the fits, and therefore not too much weight should be placed on its temperature dependence. In literature, the prefactor is often ignored altogether, or at least assumed to be constant with both temperature and field. Within the literature that does investigate it, a variety of different dependencies is observed $[1,5,6,17,18]$.

Several extensions of the analytical model are possible but beyond the scope of this work and we will therefore just briefly mention them here. Our description of $V^{*}$ in Eq. (5) is merely a first-degree approximation of reality, and beyond the used constant offset one could expect higherorder dependencies. The nucleus shape and size could also be restricted by the morphology of the material, leading to a weaker field dependency. The nucleus size can also depend on temperature, as suggested by Fig. 4(d). Finally, one could combine Eq. (6) with the concept of the Preisach distribution to obtain a distribution of switching times [12,29]. Finding out which of these factors, or other factors, are experimentally most relevant will require a richer experimental dataset than used here in view of the risk of overparametrization of the model.

\section{CONCLUSION}

To summarize, we have studied the switching kinetics in an organic ferroelectric using both a microscopic electrostatic model as well as an analytical model. The microscopic model succeeded in reproducing the overall behavior of the experiments, and while the field dependence in the simulations was stronger than in experiments, both follow the Merz law for the field dependence of the switching time. It is especially remarkable that the Merz law is obtained in a system of simple interacting dipoles as in the presented model. The Merz law is often associated with domain walls and defects, neither of which were (explicitly) included in the presented model. This suggests that the Merz law is even more general than previously assumed.

Detailed analysis of the switching process showed how the macroscopic switching time is the result of thermal activation over an energy barrier, which in turn directly relates to the process of nucleation. This insight allowed us to extend the theory of thermally activated nucleation-limited switching with the concept of a field-dependent nucleus size. The resulting model has the functional shape of the Merz law and provides a single consistent parameter set when analyzing both the macroscopic switching time and the microscopic nucleation, proving the relation between the two. The analytical model could also describe the experimental field and temperature dependence of the switching time in BTA. While doing so, it provides a natural explanation for the emergence of Merz behavior, which is generally applicable to polycrystalline ferroelectrics.

\section{ACKNOWLEDGMENTS}

We thank Professor Rint Sijbesma and Dr. Xiao Meng for providing the BTA materials. T.D.C. acknowledges financial support from the Swedish Government Strategic Research Area in Materials Science on Functional Materials at Linköping University (Faculty Grant SFO Mat LiU No. 2009 00971). I.U. acknowledges funding by Vetenskapsrådet.

\section{APPENDIX A: DIFFERENT TYPES OF DEFECTS}

To investigate the effect specific defects might have, we have simulated several different scenarios of single columns with one type of defect. Since the chirality of the helix can change at a defect, we can distinguish between chiral and nonchiral defects, as shown in Fig. 7. The rotation angle of a defect can also vary. Normally, these angles are chosen 


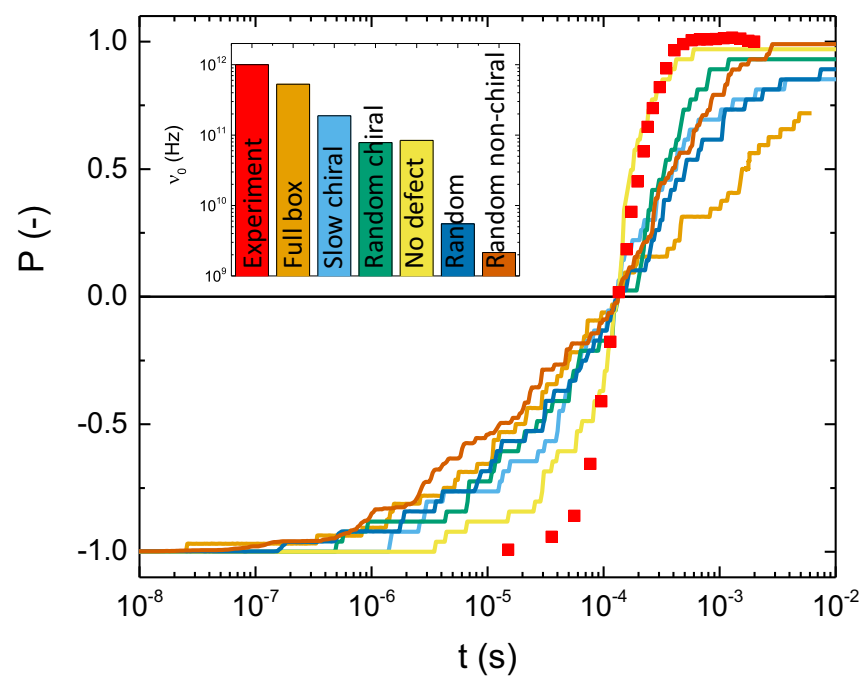

FIG. 8. Comparison of the simulated switching transients for systems with different types of defects with a transient obtained from experiment. Random refers to the rotation angle at the defects as shown in Fig. 7. The simulation results are shifted to have the same switching time as the experiment by adjusting the attempt frequency $v_{0}$, as shown in the inset. Results have been averaged over 100 independent columns of 200 molecules with a subcolumn length of $10 \pm 4$ and an applied field of $0.4 \mathrm{~V} / \mathrm{nm}$. The full box simulation was performed on a system of $8 \times 8$ interacting columns with random chiral defects.

randomly, but we have also included a scenario where the angle is fixed to give the slowest possible switching (being $\left.60^{\circ}\right)$.

The results of the simulations with all these types of defects are shown in Fig. 8. We apply a fixed external field of 0.4 $\mathrm{V} / \mathrm{nm}$, which is chosen as a trade-off between speed (lower at lower fields) and dispersion (stronger at lower fields). The timescale of the simulation results is solely determined by the attempt frequency $v_{0}$, which we have so far fixed at the expected phonon frequency of $1 \mathrm{THz}$, but deviations from this value are possible. For a better comparison between the scenarios we have therefore adjusted the attempt frequency such that all curves have the same switching time as the experiment. Corresponding attempt frequencies are shown in the inset to Fig. 8.

The cases of fully random and random nonchiral defects are both too dispersive compared to the experiment. When considering only chiral defects, either with random angles or fixed at the slowest angle of $60^{\circ}$, a moderate agreement with the experiment is obtained. The best agreement, especially in terms of dispersion, is obtained for no defects. However, this scenario is physically very unlikely, since defects are known to be present in these disordered liquid crystalline materials $[31,42,43]$. These results therefore suggest that in the experimental device, mainly chiral defects are present.

It is known that in solution the self-assembled BTA columns contain very few chiral defects due to the large energy penalty [44-46]. An argument as to why in thin-film devices one could nevertheless have mainly chiral defects goes as follows. In solution it is easy for the molecules to assemble and disassemble into the columns. This is not the case during device fabrication and conditioning, which happens in solid state under an applied field at elevated temperature and is a highly nonequilibrium process. When two columns of opposite chirality connect, they have limited freedom to dissociate again, and are stuck in place forming a chiral defect.

Furthermore, nonchiral defects are likely to disappear during device conditioning. While the columns cannot fully dissociate after forming a defect, they have some freedom to rotate. Since the energy is minimized if there is no defect, the columns will lock into place as shown in Fig. 7(a). The combination of the likely formation of chiral defects and the selective removal of nonchiral defects thus makes it likely for the experimental device to contain mainly chiral defects.

It should be noted that the results discussed so far were obtained for single isolated columns. While this case is instructive for understanding the effect of defects, in reality we have a system of many interacting columns. Letting the columns interact results in an even larger dispersion, as shown in the orange curve for a simulation box with intercolumnar interaction. This is in agreement with previous observations $[29,30]$ and increases the discrepancy between simulations and experiment in terms of dispersion.

Possible explanations for the discrepancies between the kinetic model and the experiment in terms of field dependence, dispersion and absolute timescale are discussed in Appendix B below.

\section{APPENDIX B: POSSIBLE EXPLANATIONS FOR DISCREPANCIES BETWEEN SIMULATIONS AND EXPERIMENTS}

\section{Disordered or dead interface layers}

There are several possible explanations for the discrepancy in field dependence between simulations and experiments that we will discuss here. The first possibility is the presence of dead or highly disordered layers at the interface. These layers are commonly assumed to be caused by the device fabrication process and have been observed experimentally $[19,42]$.

Highly disordered layers are implemented by having multiple defects close to the interface, resulting in a few short subcolumns at this interface. Since nucleation happens at the interface, having many defects there might slow down the polarization switching. In Fig. 9(a) we indeed see a slight slowdown of the kinetics, but far insufficient to reach agreement with experiments.

A slightly larger slowdown is obtained in a simulation with dead interface layers, shown in Fig. 9(b). These dead layers consist of dipoles that are "frozen," i.e., they cannot flip their direction. This again provides a barrier for contact nucleation, slowing down switching. Nevertheless, the dispersion and field dependence of the results remain too large compared to the experiments.

\section{Leaky layer model}

Another effect non-ferroelectric interface layers can have is to reduce the effective field over the ferroelectric layer. If the material has a finite conductivity, the effective field becomes 
(a)

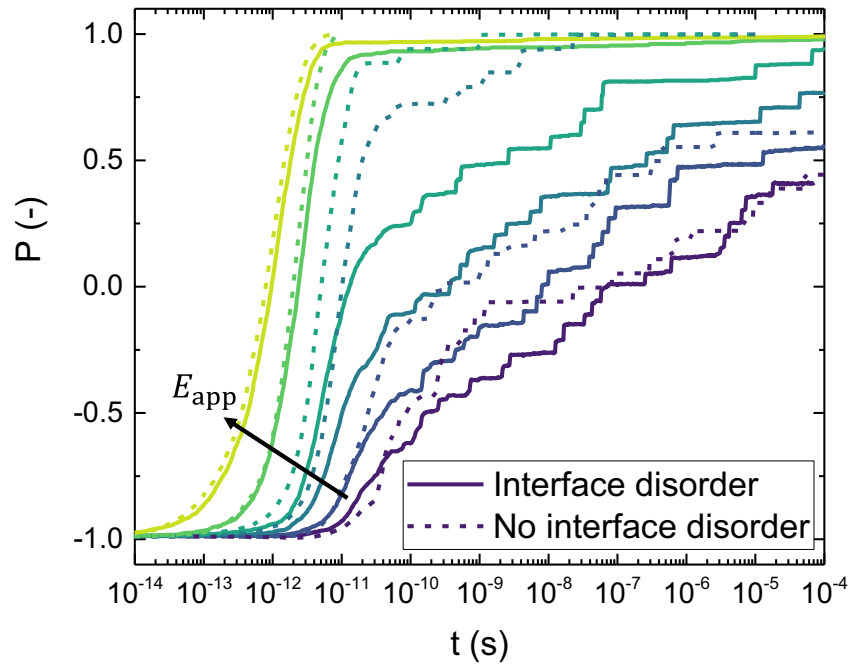

(b)

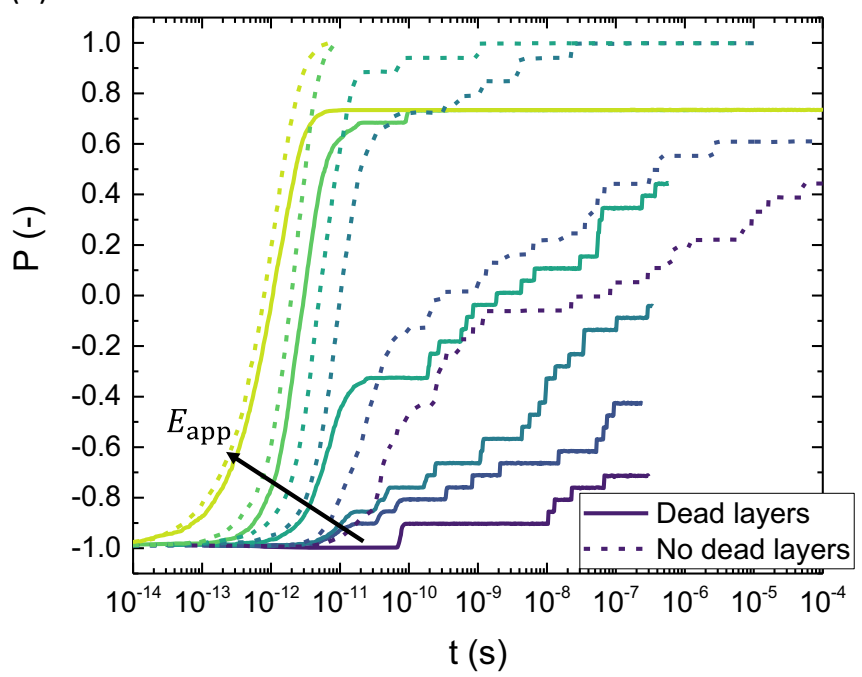

FIG. 9. Switching transients for the case of (a) interface disorder and (b) dead interface layers. The applied field is increased from 0.5 to $2 \mathrm{~V} / \mathrm{nm}$. Note that the no interface or dead layers case is the same as in Fig. S2 [33].

time-dependent which can delay the switching. This situation was first described and modeled by von Seggern and Fedosov for the case of PVDF [36,37]. Here we apply it to our system, where we use our previous simulation results as the intrinsic system response for input in the model.

This leaky layer model is schematically represented in Fig. 10(a). Following von Seggern and Fedosov, we assume our material to exist of two layers: the ferroelectric layer and a leaky dielectric layer. Taking a leaky dielectric at both interfaces with the same effective thickness would lead to equivalent results. The layers have a thickness $d$ and dielectric constant $\epsilon$, and experience a time-dependent electric field $E(t)$. Due to the conductivity $g$ of the material, a surface charge $\sigma$ will build up at the interface between the layers:

$$
\frac{d \sigma}{d t}=g\left(E_{1}-E_{2}\right)
$$

Equating the current density $j$ through the resistor $R$ and both layers, we can obtain the differential equations for the electric fields:

$$
\begin{gathered}
\frac{d E_{1}}{d t}=\frac{1}{\epsilon_{0} \epsilon_{1} A R}\left(V_{0}-\left(d_{1}+A R g\right) E_{1}-d_{2} E_{2}\right), \\
\frac{d E_{2}}{d t}=\frac{1}{\epsilon_{0} \epsilon_{2} A R}\left(V_{0}-\left(d_{2}+A R g\right) E_{2}-d_{1} E_{1}-A R \frac{d P}{d t}\right),
\end{gathered}
$$

where $A$ is the area of the device.

(b)

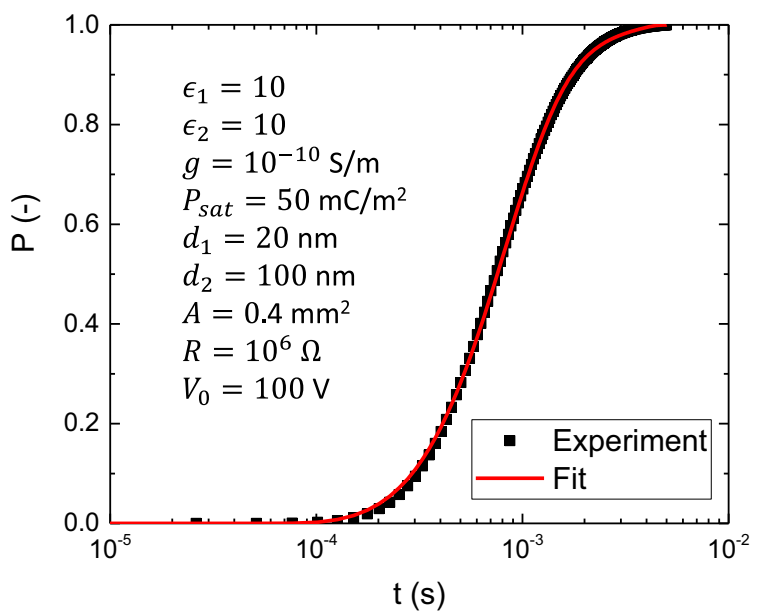

FIG. 10. The leaky layer model. (a) Schematic overview of the model with the two different layers. (b) Fit to a typical experimental switching transient using the indicated parameters. 


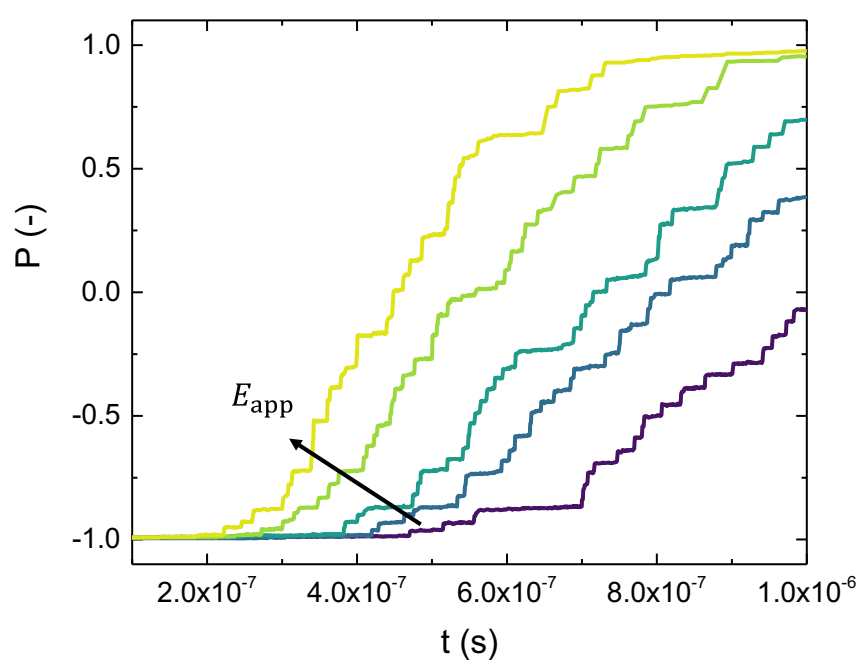

FIG. 11. Switching transients obtained in a simulation with a 1$\mu$ s field sweep. The maximum applied field is increased from 0.5 to $2 \mathrm{~V} / \mathrm{nm}$.

What is left is to obtain an expression for the change in polarization in the ferroelectric as a function of the field and polarization, $\frac{d P}{d t}\left(P, E_{2}\right)$. We use our simulations results to describe this intrinsic response and parametrize and differentiate the $P(t, E)$ curves of Fig. S2 [33]. We can now solve the equations for $\sigma, E_{1}, E_{2}$, and $P$ with the appropriate starting conditions, giving us the switching transient $P(t)$.

We fit this model to our experimental result and find a good agreement with reasonable parameters, as shown in Fig. 10(b).
The resulting switching transient is now dominated by the resistor $R$, which is set to the 1-M $\Omega$ input impedance of the oscilloscope used in experiments. When this resistance is decreased, the switching speeds up. Omitting the series resistance $R$ precludes an accurate fit, that is, the leaky dielectric alone cannot explain the discrepancy. This would imply that in our experiments, we are limited by the $R C$ time of our measurement circuit.

We perform two checks to verify if this could be the case. First, we check the effect of having a real applied voltage sweep in the simulations, instead of an instantaneous step. Our amplifier needs about $1 \mu$ s to reach a typical applied voltage. In our simulations we therefore use a linear applied field sweep from 0 to $E_{\max }$ in $1 \mu \mathrm{s}$. The results of these sweeps are shown in Fig. 11. Unsurprisingly, we see that the response becomes dominated by the sweep speed of the applied field. The switching still happens much faster than observed experimentally.

Secondly, we change our switching transient measurement setup to reduce the $R C$ time. The pulse sequence and measurement circuit are shown in Fig. 12. The switched polarization is measured as a function of the duration of the setting pulse $t_{\text {set }}$. This measurement is done using the oscilloscope with a measurement and background pulse. Any limitation on the switching speed due to the $R C$ time of the circuit would arise during the setting pulse. We therefore apply this pulse in two different configurations: the "slow" way with the oscilloscope as part of the circuit, and the "fast" way by directly connecting the ferroelectric capacitor to the ground. We find no difference between the two configurations with different $R C$ times, (a)

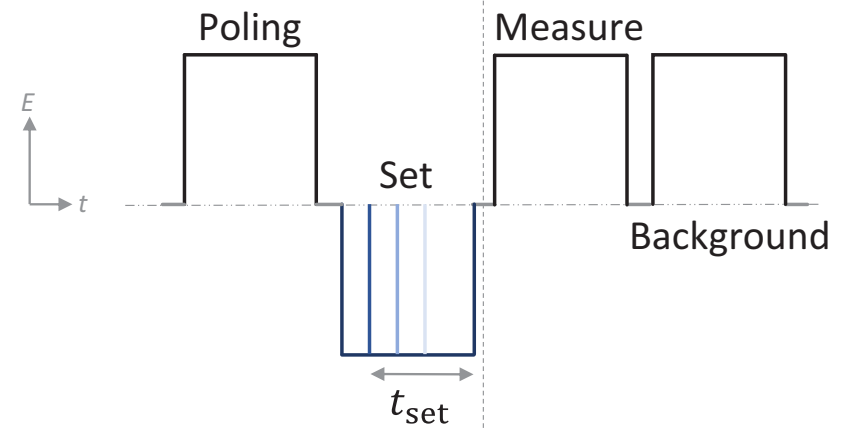

(b)

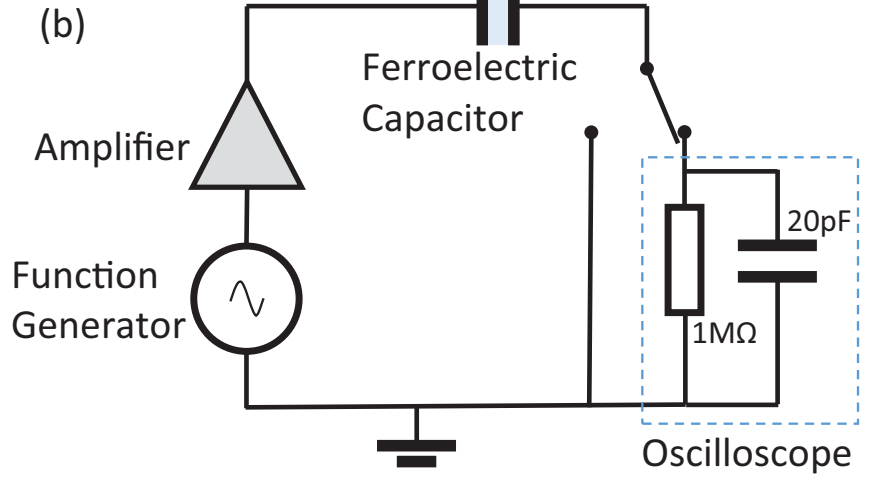

(c)

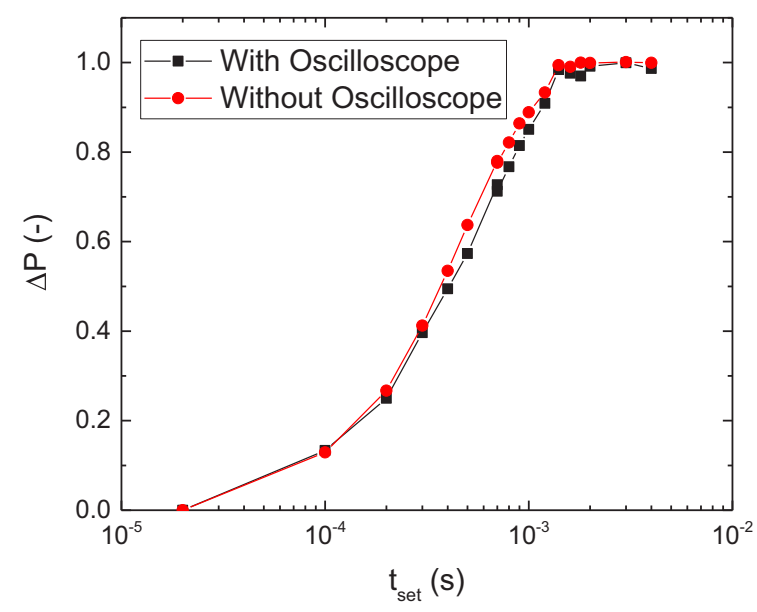

FIG. 12. Check of the experimental limitations. (a) The pulse sequence and (b) measurement circuit used to measure the switching time. (c) The switching transient measured with and without oscilloscope connected during the set pulse of length $t_{\text {set }}$. 
suggesting that the $R C$ time of the circuit is not a limiting factor in our measurement.

It thus seems unlikely that we are limited by our experimental setup in measuring switching transients. This contradicts the result of the fitting to the leaky layer model, which leads us to the conclusion that the leaky layer model cannot describe our system correctly. In addition we note that the experimentally observed field- and temperature dependencies would be hard to reconcile with a model in which the time constant is set by external factors like a series resistance $R$ associated with measurement equipment.
[1] J. Y. Jo, H. S. Han, J. G. Yoon, T. K. Song, S.-H. Kim, and T. W. Noh, Phys. Rev. Lett. 99, 267602 (2007).

[2] J. F. Scott, J. Phys.: Condens. Matter 18, R361 (2006).

[3] S. Zhukov, Y. A. Genenko, and H. von Seggern, J. Appl. Phys. 108, 014106 (2010).

[4] J. Li, B. Nagaraj, H. Liang, W. Cao, C. H. Lee, and R. Ramesh, Appl. Phys. Lett. 84, 1174 (2004).

[5] W. J. Hu, D.-M. Juo, L. You, J. Wang, Y.-C. Chen, Y.-H. Chu, and T. Wu, Sci. Rep. 4, 4772 (2015).

[6] D. Zhao, I. Katsouras, K. Asadi, P. W. M. Blom, and D. M. de Leeuw, Phys. Rev. B 92, 214115 (2015).

[7] Y. Takahashi, N. Tomoda, and T. Furukawa, Polym. J. 47, 249 (2015).

[8] H. Ishii, T. Nakajima, Y. Takahashi, and T. Furukawa, Appl. Phys. Express 4, 031501 (2011).

[9] Y. Ishibashi and Y. Takagi, J. Phys. Soc. Japan 31, 506 (1971).

[10] M. Avrami, J. Chem. Phys. 8, 212 (1940).

[11] A. N. Kolmogorov, Izv. Akad. Nauk SSSR, Ser. Mater. 3, 355 (1937)

[12] A. K. Tagantsev, I. Stolichnov, N. Setter, J. S. Cross, and M. Tsukada, Phys. Rev. B 66, 214109 (2002).

[13] A. Gruverman, B. J. Rodriguez, C. Dehoff, J. D. Waldrep, A. I. Kingon, R. J. Nemanich, and J. S. Cross, Appl. Phys. Lett. 87, 082902 (2005).

[14] O. Lohse, M. Grossmann, U. Boettger, D. Bolten, and R. Waser, J. Appl. Phys. 89, 2332 (2001).

[15] V. Shur, E. Rumyantsev, and S. Makarov, J. Appl. Phys. 84, 445 (1998).

[16] W. J. Merz, Phys. Rev. 95, 690 (1954).

[17] S. Liu, I. Grinberg, and A. M. Rappe, Nature (London) 534, 360 (2016).

[18] Y.-H. Shin, I. Grinberg, I.-W. Chen, and A. M. Rappe, Nature (London) 449, 881 (2007).

[19] A. V. Gorbunov, T. Putzeys, I. Urbanavičiūte, R. A. J. Janssen, M. Wübbenhorst, R. P. Sijbesma, and M. Kemerink, Phys. Chem. Chem. Phys. 18, 23663 (2016).

[20] Q. Meng, M.-G. Han, J. Tao, G. Xu, D. O. Welch, and Y. Zhu, Phys. Rev. B 91, 054104 (2015).

[21] A.-Q. Jiang, H. J. Lee, C. S. Hwang, and T.-A. Tang, Phys. Rev. B 80, 024119 (2009).

[22] H. Ishii, T. Nakajima, T. Furukawa, and S. Okamura, Jpn. J. Appl. Phys. 52, 041603 (2013).

[23] D. Zhao, T. Lenz, G. H. Gelinck, P. Groen, D. Damjanovic, D. M. de Leeuw, and I. Katsouras, Nat. Commun. 10, 2547 (2019).

[24] R. C. Miller and G. Weinreich, Phys. Rev. 117, 1460 (1960).

[25] T. Tybell, P. Paruch, T. Giamarchi, and J.-M. Triscone, Phys. Rev. Lett. 89, 097601 (2002).

[26] S. M. Yang, J. Y. Jo, T. H. Kim, J.-G. Yoon, T. K. Song, H. N. Lee, Z. Marton, S. Park, Y. Jo, and T. W. Noh, Phys. Rev. B 82, 174125 (2010).
[27] Y. A. Genenko, S. Zhukov, S. V. Yampolskii, J. Schütrumpf, R. Dittmer, W. Jo, H. Kungl, M. J. Hoffmann, and H. von Seggern, Adv. Funct. Mater. 22, 2058 (2012).

[28] M. Vopsaroiu, J. Blackburn, M. G. Cain, and P. M. Weaver, Phys. Rev. B 82, 024109 (2010).

[29] I. Urbanavičiūte, T. D. Cornelissen, X. Meng, R. P. Sijbesma, and M. Kemerink, Nat. Commun. 9, 4409 (2018).

[30] T. D. Cornelissen, M. Biler, I. Urbanaviciute, P. Norman, M. Linares, and M. Kemerink, Phys. Chem. Chem. Phys. 21, 1375 (2019).

[31] I. Urbanavičiūte, X. Meng, T. D. Cornelissen, A. V. Gorbunov, S. Bhattacharjee, R. P. Sijbesma, and M. Kemerink, Adv. Electron. Mater. 3, 1600530 (2017).

[32] I. Urbanavičiūte, S. Bhattacharjee, M. Biler, J. A. M. Lugger, T. D. Cornelissen, P. Norman, M. Linares, R. P. Sijbesma, and M. Kemerink, Phys. Chem. Chem. Phys. 21, 2069 (2019).

[33] See Supplemental Material at http://link.aps.org/supplemental/ 10.1103/PhysRevB.101.214301 for model details, results of an infinite column without defects, justification for ignoring intercolumnar interactions, energy scaling argument in three dimensions, and details on fitting the experimental data.

[34] N. M. Casellas, I. Urbanaviciute, T. D. Cornelissen, J. A. Berrocal, T. Torres, M. Kemerink, and M. García-Iglesias, Chem. Commun. 55, 8828 (2019).

[35] I. Urbanavičiūte, X. Meng, M. Biler, Y. Wei, T. D. Cornelissen, S. Bhattacharjee, M. Linares, and M. Kemerink, Mater. Horizons 6, 1688 (2019).

[36] S. N. Fedosov and H. von Seggern, J. Appl. Phys. 96, 2173 (2004).

[37] H. von Seggern and S. N. Fedosov, Appl. Phys. Lett. 81, 2830 (2002).

[38] P. Chauve, T. Giamarchi, and P. Le Doussal, Phys. Rev. B 62, 6241 (2000).

[39] A. Leschhorn, S. Djoumbou, and H. Kliem, J. Appl. Phys. 115, 114106 (2014).

[40] A. Leschhorn and H. Kliem, J. Appl. Phys. 121, 014103 (2017).

[41] R. H. Fowler and L. Nordheim, Proc. R. Soc. London, Ser. A 119, 173 (1928).

[42] A. V. Gorbunov, X. Meng, I. Urbanavičiūte, T. Putzeys, M. Wübbenhorst, R. P. Sijbesma, and M. Kemerink, Phys. Chem. Chem. Phys. 19, 3192 (2017).

[43] Y. Shishido, H. Anetai, T. Takeda, N. Hoshino, S. I. Noro, T. Nakamura, and T. Akutagawa, J. Phys. Chem. C 118, 21204 (2014).

[44] B. Jouvelet, B. Isare, L. Bouteiller, and P. van der Schoot, Langmuir 30, 4570 (2014).

[45] M. M. J. Smulders, I. A. W. Filot, J. M. A. Leenders, P. van der Schoot, A. R. A. Palmans, A. P. H. J. Schenning, and E. W. Meijer, J. Am. Chem. Soc. 132, 611 (2010).

[46] J. van Gestel, Macromolecules 37, 3894 (2004). 